\title{
Highly sensitive detection of thrombin using metal-waveguide-capillary based photometer and gold nanorods probe
}

Jiqing Ye ${ }^{a}$, Yue Zhang ${ }^{a}$, Jian Wang ${ }^{a,{ }^{*}}$, Hui Huang ${ }^{b,{ }^{*}}$, Zhou Jiang ${ }^{c}$, Min Bai ${ }^{b}$ a. Ministry of Education Key Laboratory of Analysis and Detection for Food Safety, Fujian Provincial Key Laboratory of Analysis and Detection for Food Safety, Fuzhou University, Fuzhou, 350108, China.

b. Department of Electronic Science and Technology, Faculty of Electronic Information and Electrical Engineering, Dalian University of Technology, Dalian 116024, China.

c. Cancer Metastasis Alert and Prevention Center, College of Chemistry; Fujian Provincial Key Laboratory of Cancer Metastasis Chemoprevention and Chemotherapy, Fuzhou University, Fuzhou 350002, China.

* Correspondence and requests for materials should be addressed to Hui Huang (Email: huihuang@dlut.edu.cn) and Jian Wang (Email: jwang@fzu.edu.cn). 


\section{Abstract}

This work described a sensitive thrombin analysis method employing metal-waveguide-capillary (MWC) photometry with a sandwich aptamergold nanorods (GNRs) as a signal transporting probe. The sandwich aptasensor consisted of a pair of thrombin-binding aptamers: one 29-mer aptamer modified with magnetic beads as capture aptamers; and one 15-mer aptamer binding with GNRs as report aptamers. Through the measurement of GNRs from the sandwich complex, the MWC photometry enabled to detect thrombin with a linear range from $0.068 \mathrm{nM}$ to $1.09 \mathrm{nM}$, and with a detection limit as low as $0.02 \mathrm{nM}$. The detection limit was improved 68 -fold $v s$. that of commercial spectrophotometer with $1 \mathrm{~cm}$ cuvette.

Key words: metal-waveguide-capillary based photometer, aptamer, gold nanorods, Thrombin 


\section{Introduction}

Thrombin is the last enzyme protease involved in the coagulation cascade, which converts fibrinogen to insoluble fibrin that forms a fibrin gel in physiological conditions or in a pathological thrombus. Having hormone-like properties, thrombin is involved in thrombosis and platelet activation. It plays a central role in a number of cardiovascular diseases [1], and regulates many processes in inflammation and tissue repair at the blood vessel wall. Picomolar thrombin in blood is known to be associated with diseases, so it is important to assess this protein at trace level with high sensitivity [2].

So far, thrombin has been highly selectively detected by aptasensors based on electrochemistry [3,4], fluorescence [5], chemiluminescence [6], surface enhanced Raman spectroscopy [7,8], quartz crystal micro-balance [9], etc. However, aptasensors have seldom been developed basing economical UV-vis absorption spectrometry method due to lower sensitivity. Metal-waveguide-capillary (MWC) based photometer is a new analytical method that has enhanced sensing performance and simplified sample preparation. The optical path length of MWC can be greatly augmented, because light can be confined inside the capillary and refracted repeatedly. For the photometer with a 7 $\mathrm{cm}$-long capillary, the detection limit can be improved 3000-fold compared with that of commercial spectrophotometer with $1 \mathrm{~cm}$ cuvette, as we previously reported [10].

Gold nanorods (GNRs) can be used as biomarkers when combined with a variety of biomolecules. They have been widely applied in medical diagnostics, medicine, environmental monitoring and other fields. A GNRs probe modified by nucleic acid aptamer that worked as a probe for transporting signal was combined with magnetic beads to separate and 
to enrich samples efficiently, and thrombin was specifically detected [11-14].

Recently, thrombin-monitoring biosensors have been prepared by a sandwich-like structure between thrombin and two aptamers for signal transformation [15-22]. Thrombin has two electropositive exosites. One is the fibrinogen-binding site and the other is the heparin-binding site. Over the past decade, two thrombin-binding aptamers (15-mer and 29-mer) have been screened out by SELEX technique. Lin et al. [23] indicated that 15-mer bound the fibrinogen-binding site of thrombin through a G-quadruplex structure dominantly via electrostatic interactions, while 29-mer bound the heparin-binding site of thrombin using a duplex structure mainly through hydrophobic effects. Daniel et al. [24] confirmed the binding of 15-mer to both heparin and fibrinogen exosites of thrombin and the selectivity of 29-mer towards the heparin-binding site. Considering that 29-mer is already selective to the heparin exosite, the formation of sandwich complex can be promoted by binding of thrombin to a second reporter aptamer, i.e. 15-mer, due to its low selectivity.

Herein, an MWC photometer was designed for thrombin detection aiming to enhance the overall analytical performance. Compared with other detection approaches, this method enjoys extremely high sensitivity, simple instrumentation, low production cost, fast response, and portability.

\section{Experimental}

\subsection{Reagents}

Thrombin, lysozyme, bovine serum albumin and bovine hemoglobin lyophilized erythrocytes were purchased from Sigma-Aldrich (USA). Synthetic anti-thrombin oligonucleotides, the 15-mer aptamer (5-(SH)- $\left(\mathrm{CH}_{2}\right)_{6}$-GGT TGG TGT GGT TGG-3) and the 29-mer aptamer 
(5-(Biotin)-( $\left(\mathrm{CH}_{2}\right)_{6}$-AGT CCG TGG TAG GGC AGG TTG GGG TGA CT-3) were obtained from Sangon Biotech (Shanghai) Co., Ltd.

Streptavidin MagneSphere $\AA$ paramagnetic particles $(1.0 \mu \mathrm{m}, 1 \%)$ were purchased from Promega (USA). All other reagents were of analytical grade. All solutions were prepared with doubly distilled water. The following buffers were used for the construction of the sandwich complex for assays: buffer A (1 mM Tris-Hcl, pH 7.4); buffer B $(20 \mathrm{mmol} / \mathrm{L}$ Tris- $\mathrm{HCl}, \quad 140 \mathrm{mmol} / \mathrm{L} \mathrm{NaCl}, \quad 5 \mathrm{mmol} / \mathrm{L} \mathrm{KCl}, 1 \mathrm{mmol} / \mathrm{L} \mathrm{MgCl} 2,1 \mathrm{mmol} / \mathrm{L}$ $\mathrm{CaCl}_{2}, 1 \mathrm{mg} / \mathrm{mL}$ BSA, $\mathrm{pH} 7.4$ ).

\subsection{Synthesis of GNRs}

GNRs were synthesized by seeded-growth method, which is currently the most widely used method [25].

Preparation of seed Solution. CTAB solution $(10 \mathrm{~mL}, 0.10 \mathrm{M})$ was mixed with $0.25 \mathrm{~mL}$ of $0.01 \mathrm{M} \mathrm{HAuCl}_{4}$. To the stirred solution, $0.60 \mathrm{~mL}$ of ice-cold $0.010 \mathrm{M} \mathrm{NaBH}_{4}$ was added, which resulted in the formation of a brownish yellow solution. Vigorous stirring of the seed solution was continued for $2 \mathrm{~min}$. Then the solution was kept at $30^{\circ} \mathrm{C}$ before further treatment.

Growth of GNRs. CTAB (100 mL, $0.10 \mathrm{M}$ ) was added to $0.1 \mathrm{~mL}$ of 0.10 $\mathrm{M} \mathrm{AgNO}_{3}$ solution at $25^{\circ} \mathrm{C}$. To this solution, $5.0 \mathrm{~mL}$ of $0.01 \mathrm{M} \mathrm{HAuCl}_{4}$ was added, $2 \mathrm{ml}$ of 12 -fold diluted hydrochloric acid was added. After gentle mixing of the solution, $0.8 \mathrm{~mL}$ of $0.10 \mathrm{M}$ ascorbic acid was added. As a mild reducing agent, ascorbic acid changed the growth solution from brownish yellow to colorless. Then $0.5 \mathrm{ml}$ of the seed solution was the added to the growth solution at $27-30{ }^{\circ} \mathrm{C}$. The mixture was violent oscillated for $20 \mathrm{~s}$, followed by standing for $24 \mathrm{~h}$ at room temperature.

2.3. The construction of MB-Apt29-thrombin-Apt15-GNRs sandwich structure for thrombin detection 
2.3.1. Preparation of 29-mer thrombin-binding aptamer-conjugated magnetic beads

$50 \mu \mathrm{L}$ of Apt-29 (10 $\mu \mathrm{mol} / \mathrm{L})$ was heated in a water bath at $90^{\circ} \mathrm{C}$ for 3 min. After cooling to room temperature, $50 \mu \mathrm{L}$ of streptavidin-coated magnetic bead suspension was added and shaken for $2 \mathrm{~h}$. Unreacted reagents were washed out using amagnetic separator and $200 \mu \mathrm{L}$ of buffer $B$ three times. Finally, the magnetic beads were re-suspended in $100 \mu \mathrm{L}$ of buffer $\mathrm{A}$.

2.3.1 Preparation of 15-mer thrombin binding aptamer-conjugated GNRs

After a solution of Apt-15 (10 $\mu \mathrm{mol} / \mathrm{L})$ was added, a thiol-modified nucleic acid aptamer was added into the centrifuge tube containing 1.5 $\mathrm{mL}$ of freshly prepared GNRs, and the mixture was allowed to stand for $16 \mathrm{~h}$ at $4^{\circ} \mathrm{C}$. GNRs were fully self-assembled. Then, $50 \mu \mathrm{l}$ of buffer solution (2 $\mathrm{mol} / \mathrm{L} \mathrm{NaCl}+50 \mathrm{mmol} / \mathrm{L}$ Tris- $\mathrm{HCl}$ ) was gradually added to the solution, and the mixture was allowed to stand and stabilized. After about $24 \mathrm{~h}$, the solution was centrifuged at $10000 \mathrm{rpm}$ for $20 \mathrm{~min}$ to separate GNRs from unbound aptamers. The supernatant was centrifuged and washed with buffer $A$, and the obtained GNRs were dispersed in $1 \mathrm{~mL}$ of buffer $\mathrm{B}$ and stored at $4^{\circ} \mathrm{C}$.

2.3.3 Preparation of MB-Apt29-thrombin-Apt15-GNRs sandwich structure

A sandwich sensor was prepared based on the signal transporting strategy. Apt29 was conjugated to magnesphere (Apt29-MB) for the later magnetic separation. Apt15 was combined with GNR (Apt15-GNRs) by thiol group and working as report aptamers.

In the strategy, $200 \mu \mathrm{L}$ of thrombin samples at various concentrations in the buffer $A$ were firstly incubated with $10 \mu \mathrm{L}$ of Apt29-MB for $10 \mathrm{~min}$ at room temperature. After that, the supernatant was removed, and the remaining magnetic beads were rinsed twice with $50 \mu \mathrm{L}$ of buffer $\mathrm{B}$. Then 
$45 \mu \mathrm{L}$ of buffer $B$ and $5 \mu \mathrm{L}$ of Apt15-GNRs at $100 \mathrm{nmol} / \mathrm{L}$ were incubated with the beads for $30 \mathrm{~min}$ at room temperature. Thus, the magnesphere/thrombin/GNRs sandwich structure was formed and followed by magnetic separation. Then the sandwich complex was washed three times with $50 \mu \mathrm{L}$ of buffer $B$ and redispersed in $150 \mu \mathrm{L}$ of $1 \%$ SDS for $30 \mathrm{~min}$ to release GNRs. The GNRs were separated as supernatant, which were then mixed with the $50 \mu \mathrm{L}$ of DMF and dilute to $1.0 \mathrm{~mL}$ test solution with deionization water.

The test solutions were transferred into the MWC photometer or the commercial spectrophotometer for extinction detection. The as prepared solution without the addition of thrombin was used as the test blank solution. Deionization water were used as the solvent control.

\subsection{Apparatus}

The MWC based photometer was assembled as previously reported [10]. As shown in Fig. 1, consists of a $7 \mathrm{~cm}$-long MWC (1.7 mm i.d., 3.18 $\mathrm{mm}$ o.d., EP-grade electropolishing inner surface, SUS316L stainless steel capillary), a $505 \mathrm{~nm}$ LED (Thorlabs M505F1), a gain tunable photodetector (Thorlabs PDB450C) with a lens, and two T-connectors used for optical coupling and fluid inlet/outlet. A three-way valve connected to the inlet Peek pipe is used to switch the inflow samples. The peek pipe is close to the quartz plate and MWC, so the dead volume in the T-connector can be minimized to effectively avoid gas bubble trapping. Moreover, the collimated light beam can be easily and efficiently coupled into MWC through the quartz plate of T-connector.

The light beam and the liquid sample were introduced into MWC by the T-connector, and the beam transmission through MWC was received by the photodetector. The inflow solution of test solution was introduced alternately into MWC via the three-way valve.

For comparison, a commercial spectrophotometer (Agilent Technologies Cary 300 Series, equipped with high performance R928 photomultiplier tube) 
with $1.0 \mathrm{~cm}$ cuvette cell was employed to measure the absorbance/extinction of the test solutions.

\section{Results and discussion}

3.1. Extinction spectra of GNRs suspension by UV-vis spectrophotometer

Fig. 2 shows a TEM image of a GNRs suspension. The average diameter, length, and aspect ratio of the starting nanorods are $(12.1 \pm 3.0) \mathrm{nm}$, $(48.8 \pm 6.3) \mathrm{nm}$, and $4.0 \pm 1.2 \mathrm{~nm}$, respectively. The average aspect ratio value is in accordance with the values found in the literature for GNRs of the same origin $[26,27]$.

The GNRs solution exhibits strong extinction in the visible to infrared wavelength range and is proportional to the GNRs concentration. The wavelength of the longitudinal plasmon peak centered at $830 \mathrm{~nm}$ has directly link to the aspect ratio of the resultant GNRs. We therefore use optical density (O.D., extinction per $1 \mathrm{~cm}$ at the plasmon resonance wavelength) to indicate the amount of the active ingredient GNRs in the synthesis products. The optical densities are measured by the UV-vis spectrometer. Our original GNRs solution obtained as described on section 2.2 usually have their optical densities of 2 3, corresponding to a gold mass concentration around $50 \mathrm{ppm}$. As shown in Fig. 3, GNRs present extinction peaking at 520 and $830 \mathrm{~nm}$, which is diluted ten-fold from the original solution by deionized water. Both peaks result from transverse and longitudinal surface plasmon resonances.

If there is no matrix effect, the detected response must be a linear function of analyte concentration which passes through pass through the zero point. But such ideal circumstance was invalid. Notably, even the test-blank solution which was prepared without the addition of thrombin exhibited extinction of GNRs (inset of Fig.3). So, the background correction must be performed using the as prepared test-blank solution.

3.2. Extinction spectra of GNRs suspension by MWC photometry

As suggested by the Bear-Lambert law, increased pathlength 
enhances extinction, which in turn favors the detection of weak extinction. The optical pathlength of MWC can be greatly augmented by repeated refraction. The test patterns are shown in Fig. 4 and 5. Clearly, the voltage decreased when deionization water was switched to test sample, and vice versa (Fig. 4, Tab.1). $\mathrm{V}_{\text {test }}, \mathrm{V}_{\text {water }}$ and corresponding $\Delta \mathrm{V}$ are shown in the enlarged inset of Fig. 4.

According to the Beer's law, the extinction of colored sample can be calculated by Eq. $1[10,28]$.

$$
E_{M w C}=-\log \left[\left(V_{\text {test }}-V_{\text {dark }}\right) /\left(V_{\text {water }} V_{\text {dark }}\right)\right]
$$

Where $V_{\text {dark }}$ is the background signal of the photodetector when LED is turned off. The $\mathrm{V}_{\text {dark }}$ value is $2.9 \mathrm{mV}$. $\mathrm{V}_{\text {test }}$ and $\mathrm{V}_{\text {water }}$ are the voltage signals of the test solutions and deionization water, respectively. $V_{\text {test }}$ and $V_{\text {water }}$ of each sample are the average taken over at least three cycles of test/water sequence. The output signal variation $\Delta \mathrm{V}\left(\mathrm{V}_{\text {thrombin }}\right)=\mathrm{V}_{\text {test }}-\mathrm{V}_{\text {water }}$ is obtained as showed in Fig. 4.

Actually, the response showed in Fig. 4 was induced by the blank test solution. The response value of zero concentration equals $V_{\text {thrombin }} 1.25$ $\mathrm{mV}$. In other words, the nonspecific binding between GNRs and magnesphere limited the improvement of detection limit as a result of previous sample preparation steps.

3.3. Properties of the light extinction between MWC-based photometer and commercial UV-vis spectrophotometer.

Fig. 5 shows the detected signal curves of test solutions prepared with thrombin sample concentration ranging from $1.09 \mathrm{nM}$ (Fig. 5, a) to $0.0 \mathrm{nM}$ (Fig. $5, h)$. According to Eq. 1, the $E_{M W c}$ can be calculated from the measured $V_{\text {test }}$, $V_{\text {water }}$ and $V_{\text {dark. }}$. The measurement results were listed in Table 1.

As shown in Table 1, the measured $\Delta \mathrm{V}_{2}\left(\mathrm{~V}_{\text {thrombin,2 }}\right)$ for $0.068 \mathrm{nmol} / \mathrm{L}$ thrombin is only $1.40 \mathrm{mV}$, and $\Delta \mathrm{V}_{1}\left(\mathrm{~V}_{\text {thrombin,1 }}\right)$ of test-blank sample is only $1.25 \mathrm{mV}$. The difference between the voltages of test-blank and test 
samples $\left(\Delta \mathrm{V}_{2}-\Delta \mathrm{V}_{1}\right)$ was $0.15 \mathrm{mV}$, which was nearly 10 times of the noise value $(0.016 \mathrm{mV})$. Thus, $0.068 \mathrm{nmol} / \mathrm{L}$ was regarded as the limit of quantification (LOQ) for this method, and the detection limit of MWC based photometer was evaluated as $0.02 \mathrm{nmol} / \mathrm{L}$ by 3 times of the noise value.

The extinction-concentration relationship by using the MWC based photometer is plotted in Fig.6. The equation of calibration curve (intercept is fixed at zero) is: $\Delta \mathrm{E}_{\mathrm{MWC}}=2.21 \times 10^{-4} \mathrm{C}(\mathrm{nmol} / \mathrm{L})$, with the linear range of $0.068 \sim 1.09 \mathrm{nmol} / \mathrm{L}(\mathrm{k}=0.971)$.

For comparison, the same set of samples were also measured by UV-vis spectrophotometer. In general, the photometric method is seldom employed for trace analysis for its relative low sensitivity, which suffers from reduced signal-to-noise ratio (SNR) with decreasing concentration of samples. As the extinction spectra shown (inset of Fig. 3), even at the most sensitive wavelength $830 \mathrm{~nm}$, LOQ was about $1.09 \mathrm{nmol} / \mathrm{L}$, with SNR value of 10 . As to $505 \mathrm{~nm}$, at which gold nanorods has much smaller extinction coefficient than that at $830 \mathrm{~nm}$, the baseline noise strongly reduced accuracy. Actually $E_{\text {cuvette }}$ at $505 \mathrm{~nm}$ of samples cannot be discriminated from each other.

As shown in Fig. 7, the calibration curve and limit of detection were obtained by quantification of higher concentration of test solutions using commercial UV-vis spectrophotometer at $505 \mathrm{~nm}$. The equation of calibration curve is: $E_{\text {cuvette }}=2.52 \times 10^{-3} \mathrm{C} /(\mathrm{nmol} / \mathrm{L})+0.0011$, with the linear range of $1.36 \sim 21.8 \mathrm{nmol} / \mathrm{L}(\mathrm{k}=0.998)$. The detection limit was evaluated as $1.36 \mathrm{nmol} / \mathrm{L}$ by 3 times of the noise value. So, the detection limit of MWC based photometer was improved 68-fold, compared with commercial cuvette based spectrophotometer.

A few groups reported several aptamer-based sandwich assays for thrombin by applying various detection methods like electrochemistry, ECL, surface plasmon resonance and fluorescence [23-28]. This present work showed the 
MWC-based photometer has comparable sensitivity with the literatures. Furthermore, it is reasonable to assume that the detecting sensitivity should be greatly increased with the $830 \mathrm{~nm}$ light source.

\section{Conclusions}

An MWC based photometer was applied for ultrasensitive detection of thrombin sample, coupling with the amplify strategy using GNRs. The optical pathlength was evidently increased to be much longer than the physical length of MWC, because the light scattered by the rippled and smooth metal sidewall was confined inside the capillary regardless of the incident angle. For the photometer with a $7 \mathrm{~cm}$-long MWC, the detection limit was improved 68 -fold $v s$. that of commercial spectrophotometer with $1 \mathrm{~cm}$ cuvette. Hence, as low as $0.02 \mathrm{nM}$ thrombin was still detectable.

Furthermore, the analytical performances can be improved by numerous strategies. For example, the volume of sample can be significantly decreased by the flow injection mode. Secondly, detecting with $830 \mathrm{~nm}$ rather than $505 \mathrm{~nm}$ light source can improve the limit of detection in the longitudinal mode, thus elevating the absorption of GNRs at $830 \mathrm{~nm}$ wavelength.

This compact and low-cost photometer may be widely applied in life science and environmental monitoring for trace analysis. It may exert the largest impact on bioprocess discovery at the cellular and molecular levels. In addition, optofluidics-related studies have been motivated.

\section{Acknowledgements}

The authors would like to appreciate the financial support of National Science Foundation of China (No. 81273548), the Ministry of Science and Technology of China (No. 2015CB931804), the Education Ministry 
of Fujian Province of China (JA15057), and Fuzhou University (2014-XQ-8).

\section{Reference}

1 M.T. Stubbs, W. Bode, A player of many parts: the spotlight falls on thrombin's structure. Thromb. Res., 1993, 69:1-58.

2 S. Centi, S. Tombelli, M. Minunni, M. Mascini, Aptamer based detection of plasma proteins by an electrochemical assay coupled to magnetic beads. Anal. Chem. 2007, 79:1466-1473.

3 X.X. Li, L.H. Shen, D.D. Zhang, H.L. Qi, Q. Gao, F. Ma, C.X. Zhang, Electrochemical impedance spectroscopy for study of aptamer thrombin interfacial interactions, Biosens. Bioelectron. 2008, 23:1624-1630.

4 F. Lisdat, D. Schafer, The use of electrochemical impedance spectroscopy for biosensing. Anal. Bioanal. Chem. 2008, 391:1555-1567.

5 M.N. Stojanovic, P. de Prada, D.W. Landry, Aptamer-based folding fluorescent sensor for cocaine. J. Am. Chem. Soc. $2001,123,4928-4931$.

6 Z. Jiang, T.T. Yang, M.Y. Liu, Y.L. Hu, J. Wang, An aptamer-based biosensor for sensitive thrombin detection with phthalocyanine@ $\mathrm{SiO}_{2}$ mesoporous nanoparticles, Biosens. Bioelectron., 2014,53:340-345.

7 A.R. Bizzarri, S. Cannistraro, Surface-enhanced Raman spectroscopy combined with atomic force microscopy for ultrasensitive detection of thrombin, Anal. Biochem. 2009, 393:149-154.

8 H.S. Cho, B.R. Baker, S. Wachsmann-Hogiu, C.V. Pagba, T.A. Laurence, S.M. Lane, L.P. Lee, J.B. Tok, Aptamer-based SERS sensor for thrombin detection. Nano Lett. 2008, 8:4386-4390. 
9 G.S. Bang, S.Y. Cho, B.G. Kim, A novel electrochemical detection method for aptamer biosensors, Biosens. Bioelectron. 2005, $21: 863-870$.

10 M. Bai, H. Huang, J. Hao, J. Zhang, H. Wu, B. Qu, A compact photometer based on metal-waveguide-capillary: application to detecting glucose of nanomolar concentration. Scientific reports. 2015, 5:10476.

11 Q. Xue, Z.G. Liu, Y.J. Guo, S.J. Guo. Cyclodextrin functionalized graphene-gold nanoparticle hybrids with strong supramolecular capability for electrochemical thrombin aptasensor. Biosens. Bioelectron., 2015,68:429-436.

12 X.L. Zhao, S. Li, L.G. Xu, W. Ma, X.L. Wu, H. Kuang, L.B. Wang, C.L. Xu. Up-conversion fluorescence "off-on" switch based on heterogeneous core-satellite assembly for thrombin detection. Biosens. Bioelectron.,2015,70:372-375.

13 L.P. Lu, J. Li, T.F. Kang, S.Y. Cheng. Bi-functionalized aptasensor for ultrasensitive detection of thrombin. Talanta. 2015,138(1) : 273-278.

14 L.Y. Chen, Y.G. Bao, J. Denstedt, J. Zhang. Nanostructured bioluminescent sensor for rapidly detecting thrombin. Biosens. Bioelectron., 2016, 77: 83-89.

15 L. C. Bock, L. C. Griffin, J. A. Latham, E. H. Vermaas, J. J. Toole, et a1. Selection of single stranded DNA molecules that bind and inhibit human thrombin. Nature, 1992, 355(6360): 564-566.

16 D. M. Tasset, M. F. Kublik, W. Steiner. Oligonucleotide inhibitor of human thrombin that bind distinct epitopes. J. Mol. Biol., 1997,272(5):688-698.

17 Y.F. Bai, F. Feng, L. Zhao, C.Y. Wang, H.Y. Wang, M,Z. Tian, J. Qin, Y.L. Duan, X.X. He. Aptamer/thrombin/aptamer-AuNPs sandwich enhanced surface plasmon resonance sensor for the detection of subnanomolar thrombin. Biosens. Bioelectron. 2013, 47:265-270 
18 A. Numnuam, K.Y. Chumbimuni-Torres, Y. Xiang, R. Bash, P. Thavarungkul, P. Kanatharana, E. Pretsch, J. Wang, E. Bakker. Aptamer-based potentiometric measurements of proteins using ion-selective microelectrodes. Anal. Chem. 2008, 80:707-712

19 H. Yang, J. Ji, Y. Liu, J. Kong, B. Liu, An aptamer-based biosensor for sensitive thrombin detection. Electrochem. Commun. 2009,11:38-40

$20 \mathrm{H}$. Huang, J.J. Zhu, DNA aptamer-based QDs electrochemiluminescence biosensor for the detection of thrombin. Biosens. Bioelectron. 2009, 25:927-930

21 C. Ding, Y. Ge, J.M. Lin, Aptamer based electrochemical assay for the determination of thrombin by using the amplification of the nanoparticles. Biosens. Bioelectron. 2010, 25:1290-1294

22 Y.H. Tennico, D. Hutanu, M.T. Koesdjojo, C.M. Bartel, V.T. Remcho, On-chip aptamer-based sandwich assay for thrombin detection employing magnetic beads and quantum dots. Anal. Chem. 2010,82:5591-5597

23. P.H. Lin, R.H. Chen, C.H. Lee, W.Y. Chen. Studies of the binding mechanism between aptamers and thrombin by circular dichroism, surface plasmon resonance and isothermal titration 16 . calorimetry.Colloids and surfaces B: Biointerfaces 2011,88(2):552-558.

24. C. Daniel, F. Melane, Y. Roupioz T. Livache, A. Buhot. Realtime monitoring thrombin interactions itsaptamers: Insights sandwich complex formation. Biosens. Bioelectron. 2013,40(1):186-192.

25 B. Nikoobakht and M.A. El-Sayed, Preparation and growth mechanism of gold nanorods (NRs) using seed-mediated growth method. Chem. Mater., 2003, 15:1957-1962.

26 T. A. El-Brolossy, T. Abdallah, M. B. Mohamed et al., Shape and size dependence of the surface plasmon resonance of gold 
nanoparticles studied by Photoacoustic technique. European Physical Journal: Special Topics, 2008,153(1):361-364.

27 W. Ni, X. Kou, Z. Yang, and J. Wang,Tailoring longitudinal surface plasmon wavelengths, scattering and absorption cross sections of gold nanorods. ACS Nano, 2008,2(4):677-686.

28 J.Z. Pan, B.Yao, Q. Fang, Hand-held photometer based on liquid-core waveguide absorption detection for nanoliter-scale samples. Anal. Chem., 2010,82:3394-3398. 
Table 1. Measurement results of thrombin samples

\begin{tabular}{lllclll}
\hline Num. & $\begin{array}{l}\mathrm{C} \\
(\mathrm{nmol} / \mathrm{L})\end{array}$ & $\begin{array}{c}\mathrm{V}_{\text {test }} \\
(\mathrm{mV})\end{array}$ & $\begin{array}{c}\mathrm{V}_{\text {water }} \\
(\mathrm{mV})\end{array}$ & $\begin{array}{l}\Delta \mathrm{V} \\
(\mathrm{mV})\end{array}$ & $\mathrm{E}_{\mathrm{MWC}}$ & $\Delta \mathrm{E}_{\mathrm{MWC}}$ \\
\hline 1 & 0 & 345.10 & 346.35 & 1.25 & 0.00158 & \\
2 & 0.068 & 346.11 & 347.51 & 1.40 & 0.00176 & 0.00018 \\
3 & 0.136 & 347.90 & 349.60 & 1.70 & 0.00213 & 0.00055 \\
4 & 0.273 & 342.80 & 344.80 & 2.00 & 0.00254 & 0.00096 \\
5 & 0.335 & 341.21 & 343.81 & 2.60 & 0.00332 & 0.00174 \\
6 & 0.545 & 338.10 & 340.90 & 2.80 & 0.00361 & 0.00203 \\
7 & 0.680 & 332.80 & 335.90 & 3.10 & 0.00406 & 0.00248 \\
8 & 1.090 & 330.80 & 334.50 & 3.70 & 0.00487 & 0.00329 \\
\hline $\mathrm{E}_{\mathrm{MWC}}=-\log \left[\left(\mathrm{V}_{\text {test }}-\mathrm{V}_{\text {dark }}\right) /\left(\mathrm{V}_{\text {blank }}-\mathrm{V}_{\text {dark }}\right)\right], \mathrm{V}_{\text {dark }}=2.9 \mathrm{mV}, \mathrm{V}_{\text {blank }}=\mathrm{V}_{\text {test }, 1} ;$ & &
\end{tabular}
$\Delta \mathrm{V}=\mathrm{V}_{\text {test }}-\mathrm{V}_{\text {water }} ;$

Noise $=0.016 \mathrm{mV}$, When Signal $/$ Noise $=10$, limit of quantification is $0.068 \mathrm{nmol} / \mathrm{L}$. 


\section{Captions:}

Fig. 1: Schematic diagram of the MWC based photometer

Fig. 2: Extinction spectra of a ten-fold dilution solution of original GNRs by UV-vis spectrophotometer. The mass concentrations of the Au is around $5 \mathrm{ppm}$. Insert: Extinction spectra of GNRs eluted from the aptamer based sandwich (magnesphere/thrombin/GNRs), with the corresponding original $\mathrm{C}_{\text {thrombin }}$ of 0 , $0.068,0.136,0.276,0.335,0.545,0.68,1.09 \mathrm{nmol} / \mathrm{L}$, from bottom to top.

Fig. 3: The light extinction measurement of GNRs in the aptamer based sandwich complex for detection of thrombin by MWC based photometer.

Fig. 4: Measurement results of different thrombin samples detected by MWC-based photometer. $C_{\text {thrombin }}: 1.09,0.68,0.545,0.335,0.276,0.136,0.068$, Onmol/L; Corresponding to curves: a, b, c, d, e, f, g, h, respectively.

Fig. 5: The relationship between light extinction and the concentration of thrombin detected on $505 \mathrm{~nm}$ by MWC-based photometer. Cthrombin: 0, 0.068, $0.136,0.276,0.335,0.545,0.68,1.09 \mathrm{nmol} / \mathrm{L}$.

Fig. 6: The relationship between light extinction and the corresponding of thrombin concentration detected by commerial UV-vis spectrophotometer at $505 \mathrm{~nm}$. $\mathrm{C}_{\text {thrombin: }}$ 1.36, 2.76, 5.46, 6.70, 10.9, 13.6 and $21.8 \mathrm{nmol} / \mathrm{L}$. 
Fig. 1
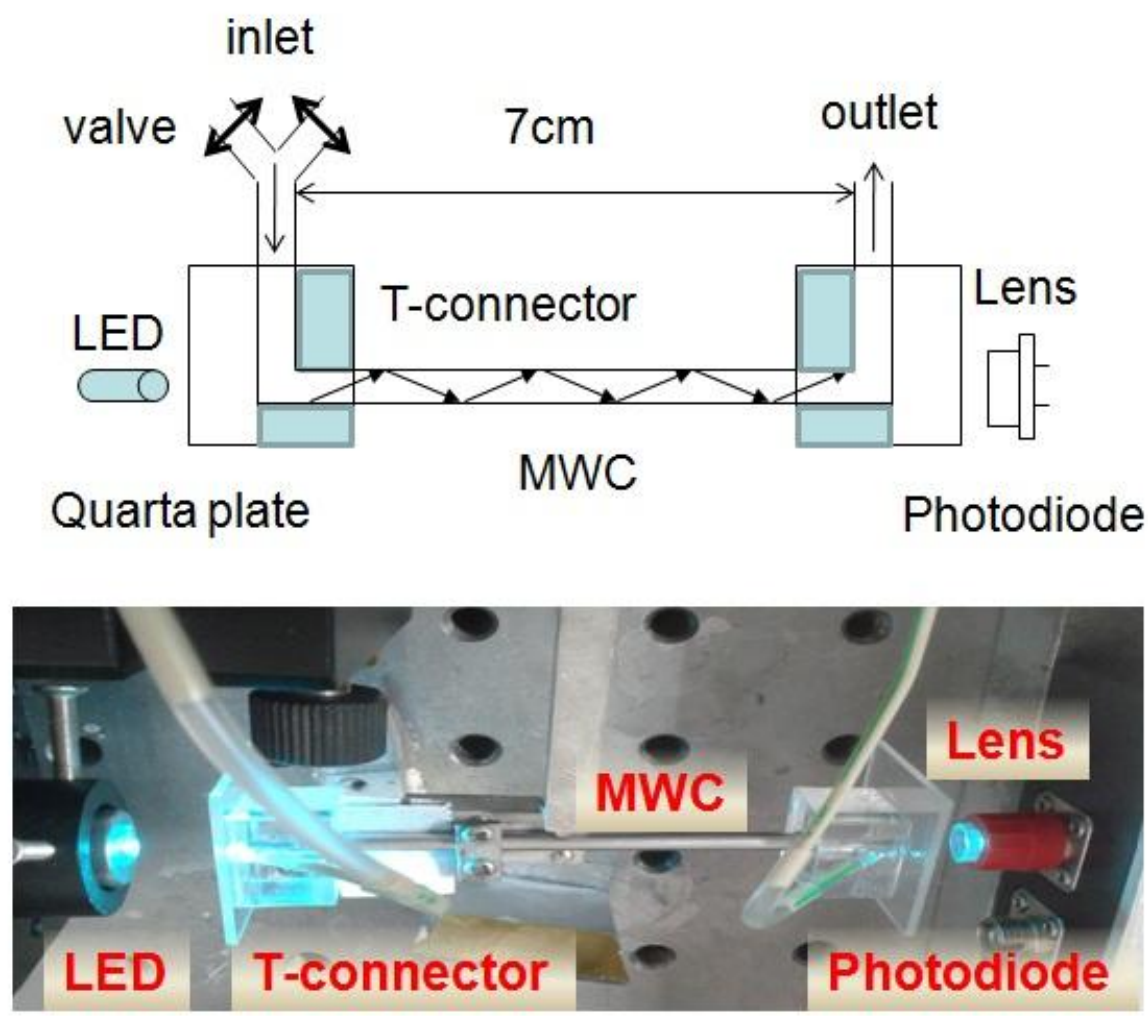
Fig. 2

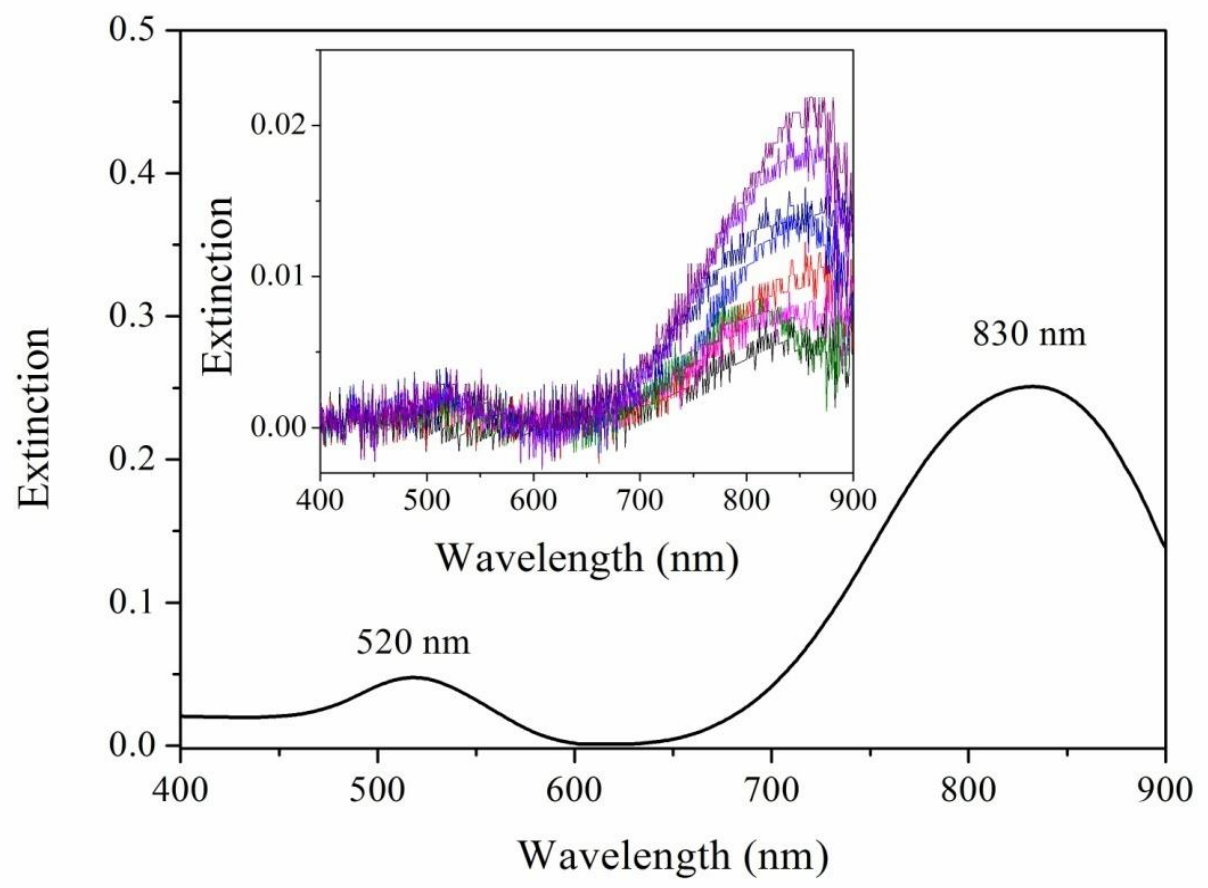


Fig. 3

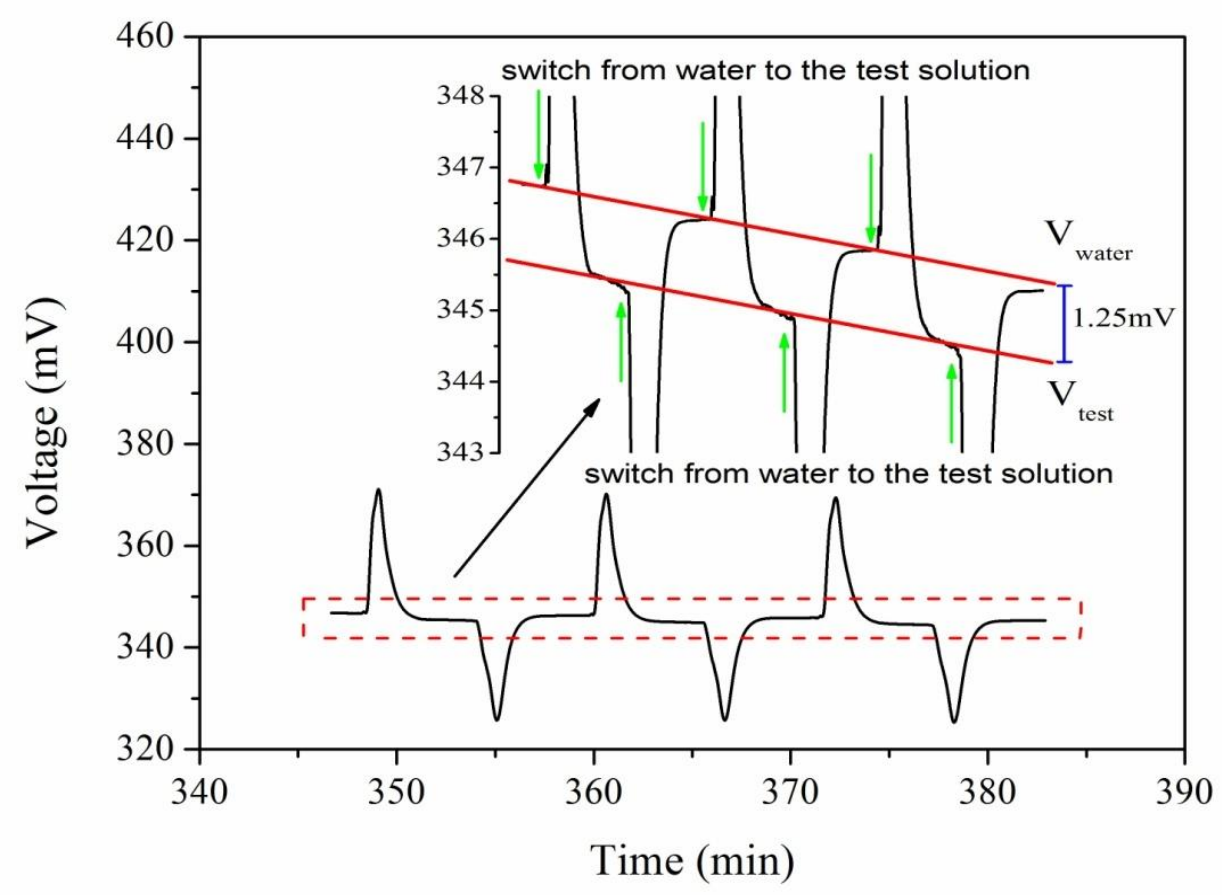


Fig. 4
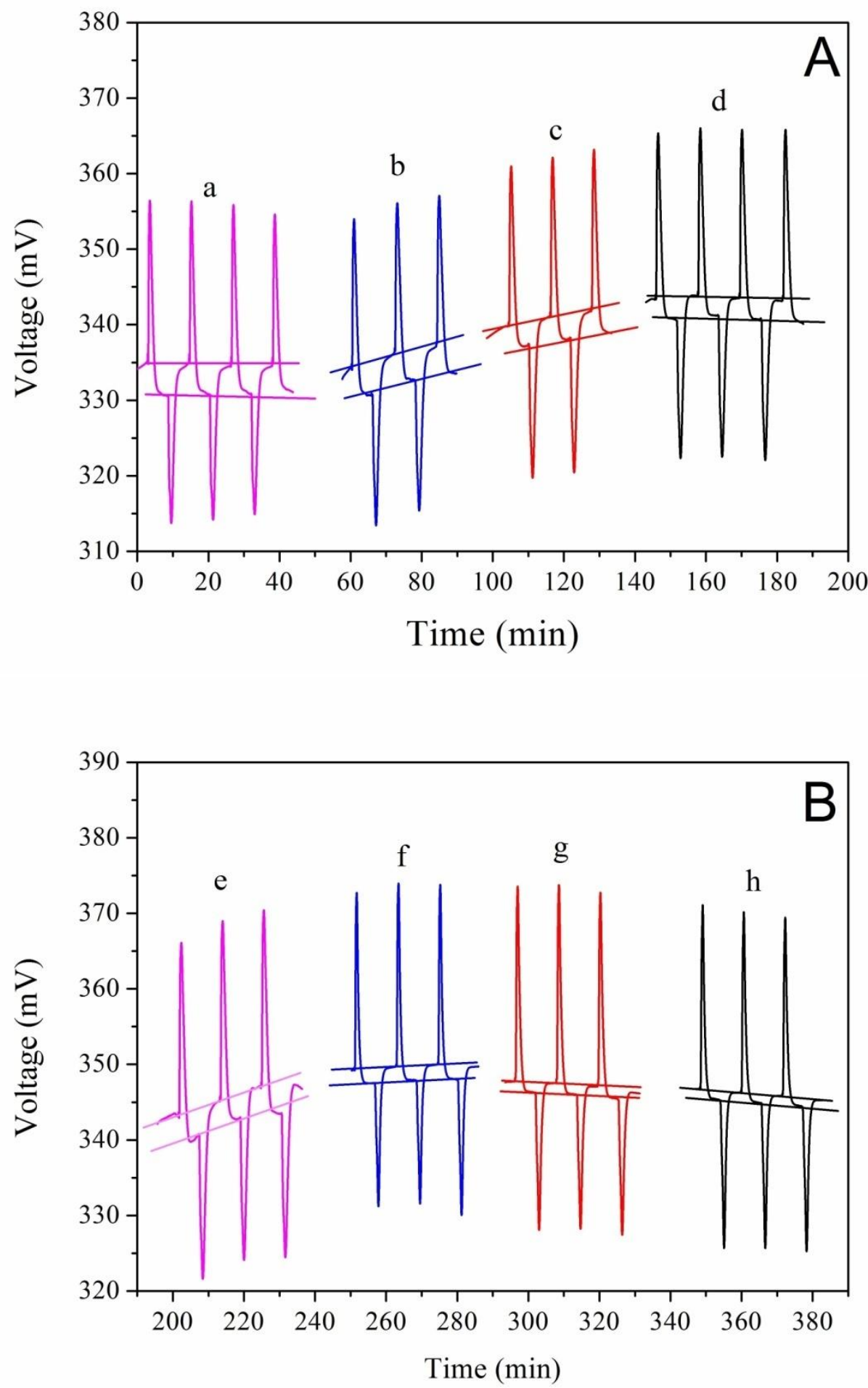
Fig. 5

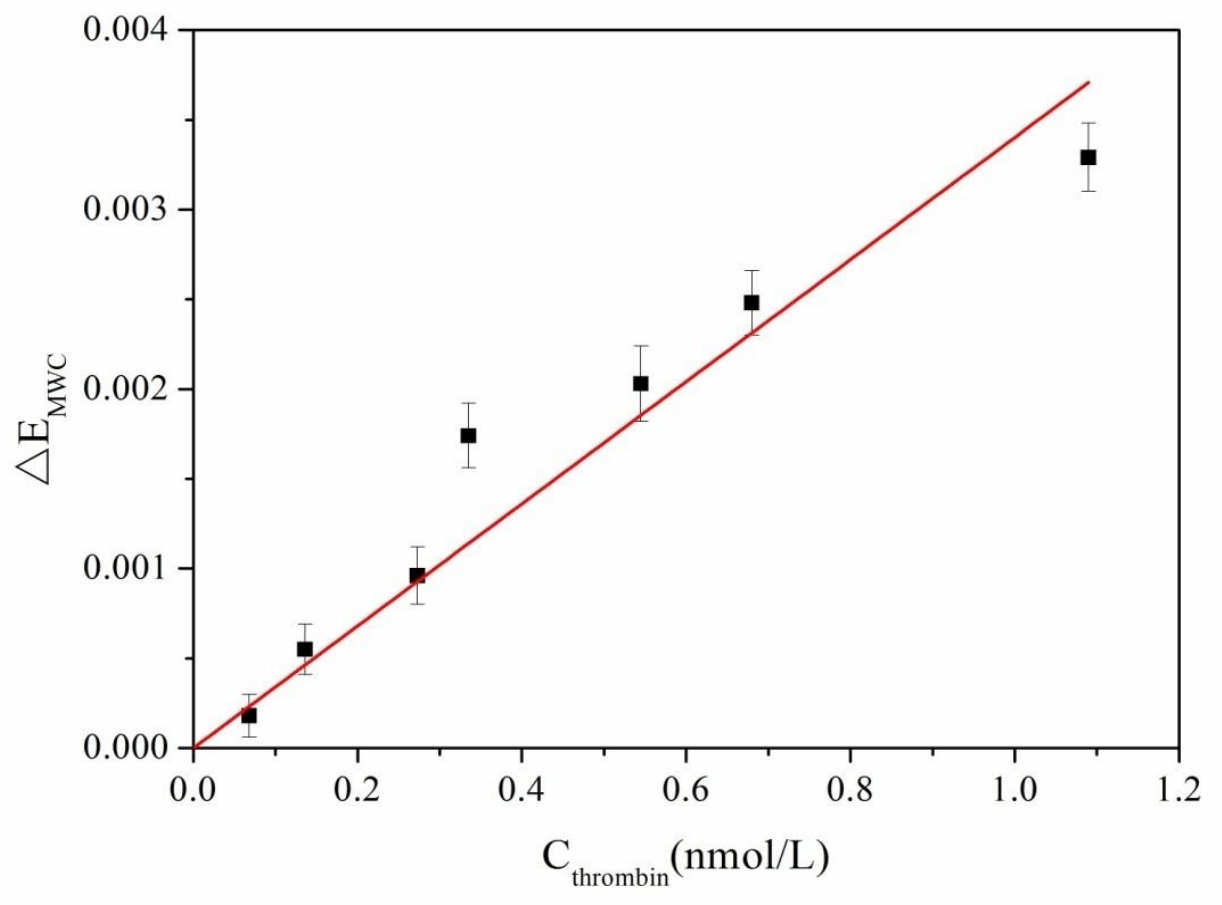


Fig.6

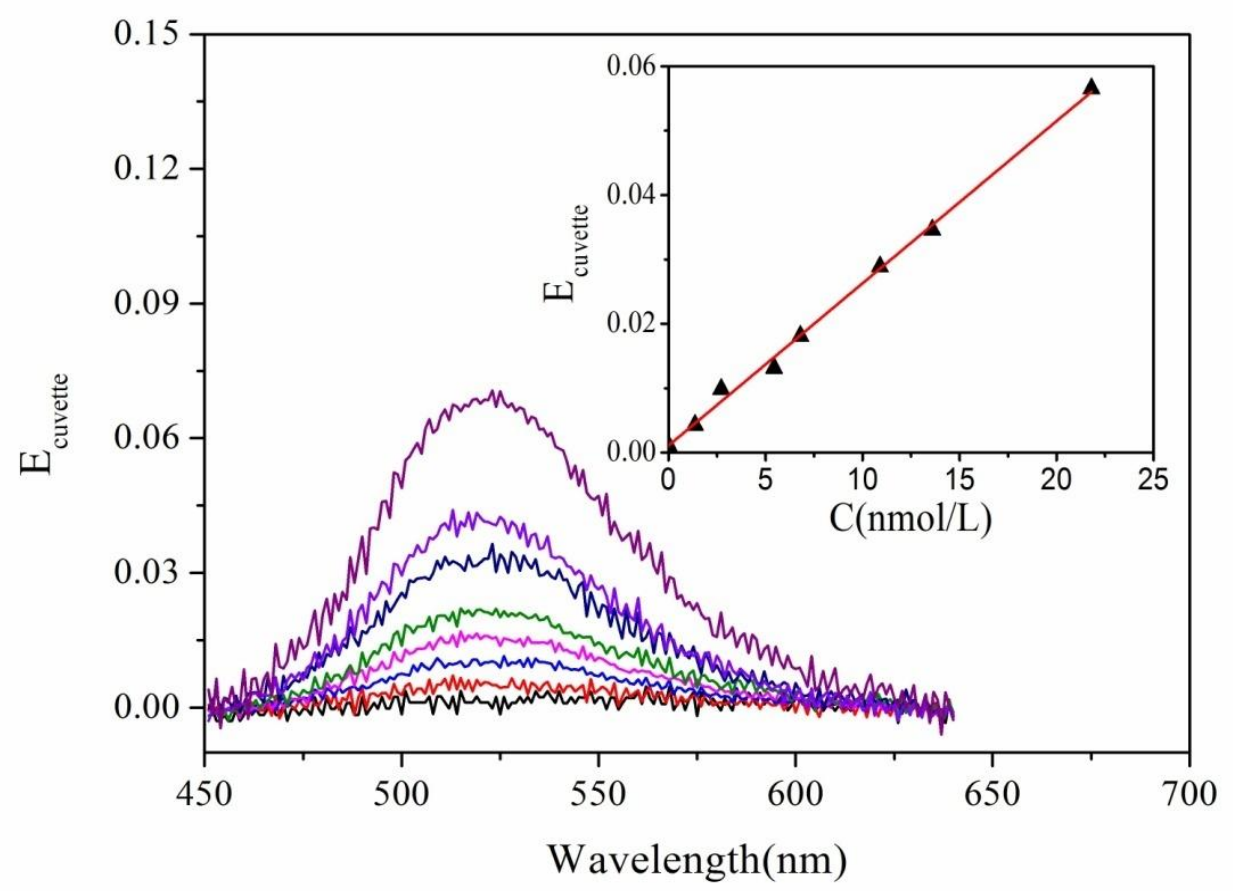

